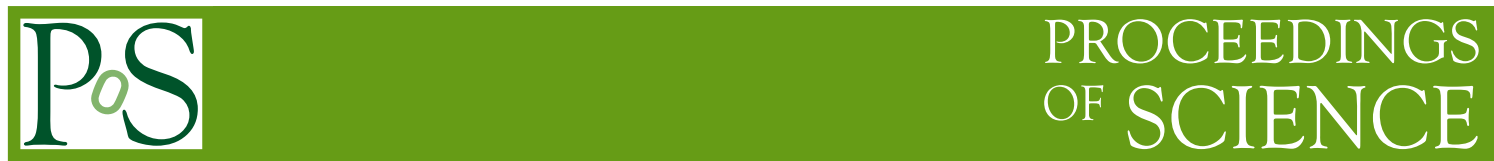

\title{
New Physics Phenomena and F-theory GUTs
}

\section{George K. Leontaris*}

Physics Department, University of Ioannina, GR-45110 Ioannina, Greece

E-mail: leonta@uoi.gr

In this presentation the new physics implications of the $B$-meson decay anomalies, observed at $\mathrm{LHCb}$, are discussed. In the first part of the talk a brief overview of the experimental status is presented. In the second part, a class of semi-local F-theory GUT models with additional neutral gauge bosons are proposed which are capable of accounting for the anomalous $B$-decay ratios $R_{K}$ and $R_{K^{*}}$.

Corfu Summer Institute 2017 'School and Workshops on Elementary Particle Physics and Gravity' 2-28 September 2017

Corfu, Greece

* Speaker. 


\section{Introduction}

In the Standard Model of strong and electroweak interactions (SM) the couplings of the neutral gauge boson $Z$ to lepton fields are flavour independent. The $Z$-boson couples in the same way to all three families of lepton fields since fermions $(f)$ with the same charge $Q_{f}=T_{3}+Y$ have a universal coupling $g^{Z f f}=g \cos \theta_{W} T_{3}-g^{\prime} \sin \theta_{W} Y$. As a result, the tree-level interactions conserve lepton flavour. This property of the SM gauge interactions is usually called Lepton Flavour Universality. The diagrams of two representative decays are shown in figure 1 . In the left hand side, the diagram for the process $e^{+} e^{-} \rightarrow Z \rightarrow \mu^{+} \mu^{-}$is shown where the $Z$-couplings to leptons are flavour diagonal. In the right hand side, the tree-level diagram for the decays $\tau \rightarrow \mu+\bar{v}_{\mu}+v_{\tau}$ and $\tau \rightarrow e+\bar{v}_{e}+v_{\tau}$ are drawn. Precision measurements show that the decay rates are equal which imply equality of the corresponding weak coupling strengths. Furthermore, in all experiments
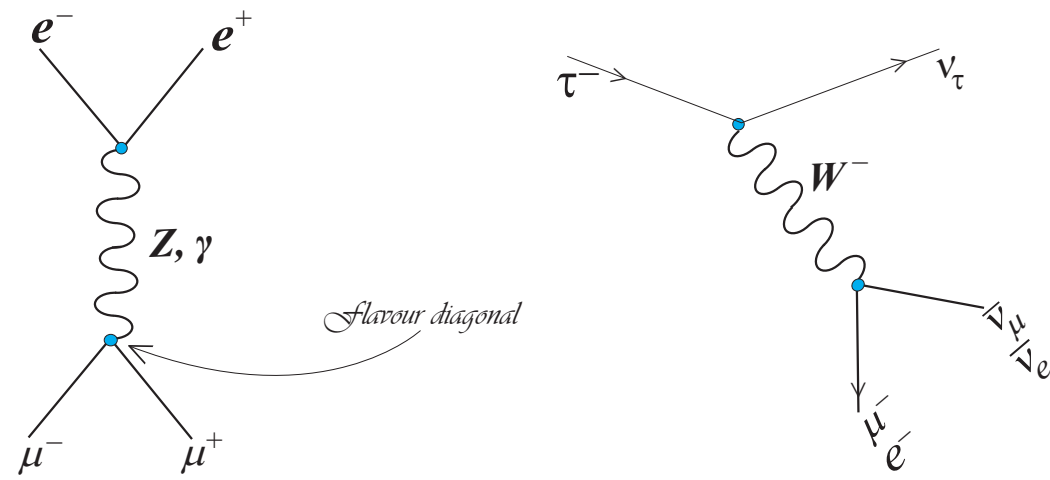

Figure 1: Representative interactions involving leptons. In the decay $e^{+} e^{-} \rightarrow Z \rightarrow \mu^{+} \mu^{-}$the $Z$ couplings are flavour diagonal in SM. In the second graph, the decay rates to the two lepton pairs $\mu \bar{v}_{\mu}$ and $e \bar{v}_{e}$ are the same. Experimentally it is found $\Gamma\left(\tau^{-} \rightarrow \mu^{-}+\bar{v}_{\mu}+v_{\tau}\right)=\Gamma\left(\tau^{-} \rightarrow e^{-}+\bar{v}_{e}+v_{\tau}\right)$.

performed during the past decades, precision tests regarding the decays of the $K$ mesons such as $K \rightarrow \ell \bar{v}_{\ell}$ where $\ell=e, \mu$, are consistent with lepton universality. However, recent experimental data from $\mathrm{LHCb}$ regarding semileptonic decays of the $B$-mesons [1, 2, 3], seem to be inconsistent with lepton universality. In the present talk we will focus on some of these processes, and in particular, those flavour changing decays involving quark fields such as $b \rightarrow s \ell^{+} \ell^{-}$where $\ell$ stand for $e, \mu, \tau$ leptons. Suitable candidates for such decays are the $B$ mesons composed of a quark-antiquark pair such as $B^{+}=\bar{b} u$ and $B^{0}=\bar{b} d$ with decay modes

$$
\begin{aligned}
B^{+} & \rightarrow K^{+} \ell^{+} \ell^{-} \\
B^{0} & \rightarrow K^{* 0} \ell^{+} \ell^{-},
\end{aligned}
$$

where $K^{+}=\bar{s} u$ and the spin- $1 K^{* 0}$ is an excited state which subsequently decays to an ordinary ground-state Kaon and a pion, $K^{* 0} \rightarrow K^{+} \pi^{-}$. Once we have analysed the present experimental data, we will propose solutions to this problem in the framework of some theoretical models inspired from F-theory. 


\section{Experimental Evidence}

We start with a few facts regarding the theoretical results obtained in the context of the Standard Model (SM), and the recent experimental evidence which is in tension with these SM predictions.

As is well known, experimentally we observe a suppression of the neutral currents compared to the charged ones involving lepton fields. For example, the branching ratios of the following two semilectonic decays of the $B$ mesons are (for related reviews see for example $[4,5,6]$ )

$$
\begin{aligned}
\operatorname{Br}\left(B \rightarrow D^{0} \ell \bar{v}\right) & =2.3 \%, \\
\operatorname{Br}\left(B \rightarrow K^{*} \ell^{+} \ell^{-}\right) & =5 \times 10^{-7},
\end{aligned}
$$

where $D^{0}=c \bar{u}$ and $K^{*}=K \pi$. The first decay proceeds with a charged intermediate gauge boson at a much larger rate compared to the second one which is mediated by neutral bosons. Focusing on the second case, we note that this is a flavour changing decay in the quark sector, $b \rightarrow s \ell^{+} \ell^{-}$ (where $\ell$ stand for $e, \mu, \tau$ leptons). In the Standard Model these flavour changing decays of the hadronic sector $(b \rightarrow s)$ proceed through one-loop graphs and are suppressed by the CKM matrix elements (see figure 2). Regarding the vertex $Z \ell^{+} \ell^{-}$, because of the universal nature of the gauge

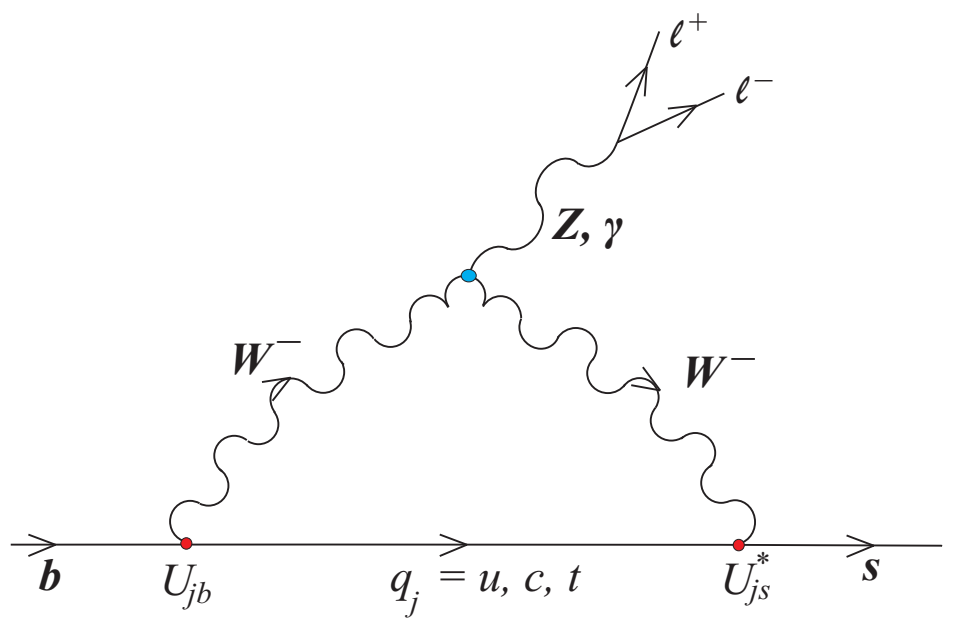

Figure 2: One-loop diagram leading to $b \rightarrow s$ flavour violation. Another contribution comes form an analogous box diagram.

couplings to leptons in the SM, both partners of the lepton pair $\ell^{+} \ell^{-}$in a given reaction are always members of the same fermion family. Moreover, the SM predictions for the branching ratios are the same for all pairs $e^{+} e^{-}, \mu^{+} \mu^{-}$and $\tau^{+} \tau^{-}$. Hence, the SM prediction for all lepton pairs for the ratio of their branching ratios is expected to be equal to unity

$$
R_{X_{i j}}=\frac{\mathrm{BR}\left(B \rightarrow X^{+} \ell_{i}^{+} \ell_{i}^{-}\right)}{\operatorname{BR}\left(B \rightarrow X^{+} \ell_{j}^{+} \ell_{j}^{-}\right)} \approx 1, i, j=e, \mu, \tau ; X=K^{+}, K^{0}, \cdots
$$


up to insignificant corrections. The experimental determination of the ratio $R_{X}$ is preferable because all theoretical uncertainties stemming from the hadronic part in the branching ratios cancel out in the ratios.

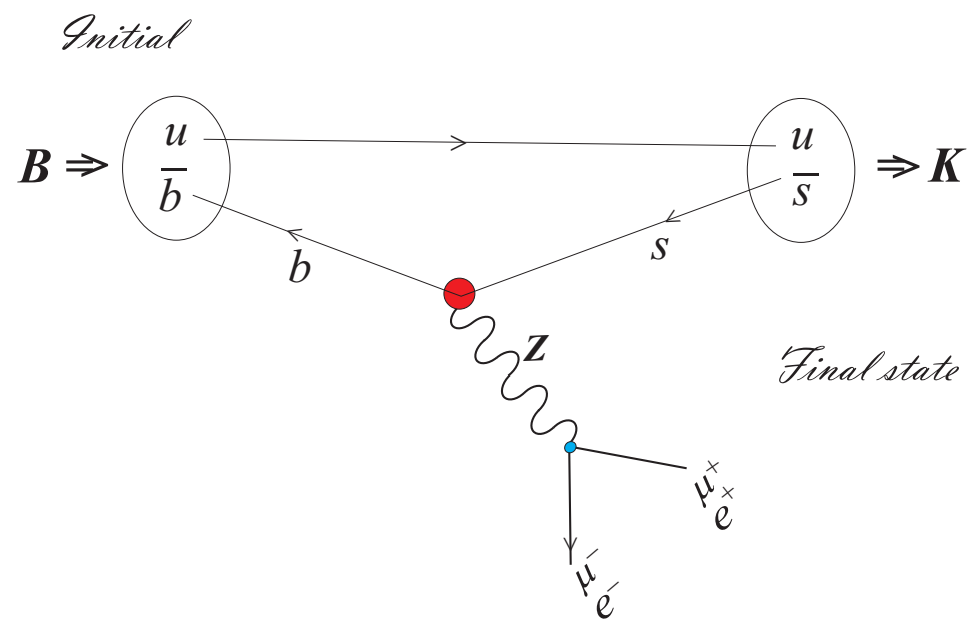

Figure 3: An illustration of the $B \rightarrow K \ell_{i}^{+} \ell_{i}^{-}$decay in SM. The red bullet stands for the one-loop contribution in figure 2.

Such decays, however, are very sensitive to contributions from interactions violating lepton flavour universality. In many extensions of the Standard Model new particles are predicted (such as neutral gauge bosons and leptoquarks) which can enhance or reduce the decay rates and modify the angular distribution of the products of the above processes. The decay (1.2), in particular, involves four particles in the final state and allows for a precise angular reconstruction in several observables. In the muon channel for example, the experiment can measure the polarization of $K^{*}$ and the dimuon pair, as well as the invariant mass square $q^{2}$, the angle $(\mu, K)$, etc.

Interestingly, recent reports [2] from the LHC experiments on the branching ratios of Bdecays to strange hadrons and lepton pairs have displayed deviations form lepton universality. The ratio of the branching ratios $B \rightarrow K^{+} \ell \bar{\ell}$ for $\ell=\mu$ or $e$, measured in the LHCb experiment is

$$
R_{K}=\frac{\mathscr{B}\left(B \rightarrow K^{+} \mu^{+} \mu^{-}\right)}{\mathscr{B}\left(B \rightarrow K^{+} e^{+} e^{-}\right)}=0.745 \pm 0.09(\text { stat }) \pm 0.036(\text { syst })
$$

where "(stat)", "(syst)" indicate statistical and systematic uncertainties respectively. This measurement is 2.6 standard deviations below the SM prediction. The decay rate of this reaction is integrated over the range of the squared dilpeton invariant mass $q^{2}$ and the region is taken to be $1 \mathrm{GeV}^{2}<q^{2}<6 \mathrm{GeV}^{2}$, which is away from the resonance region $B^{+} \rightarrow J / \psi\left(\ell^{+} \ell^{-}\right) K^{+}$, in order to have a clear experimental signature to compare with the theoretical predictions. 
Similarly, the experimental result on the ratio of branching ratios $B \rightarrow K^{*} \ell \bar{\ell}$ is given by

$$
R_{K^{*}}=\frac{\mathscr{B}\left(B \rightarrow K^{*} \mu^{+} \mu^{-}\right)}{\mathscr{B}\left(B \rightarrow K^{*} e^{+} e^{-}\right)} \approx\left\{\begin{array}{c}
0.660 \begin{array}{c}
+0.110 \\
-0.070 \\
0.685 \\
+0.113 \\
-0.069
\end{array} \pm 0.024
\end{array}\right.
$$

for the ranges $\left(2 m_{\mu}\right)^{2}<q^{2}<1.1 \mathrm{GeV}^{2}$ and $1.1 \mathrm{GeV}^{2}<q^{2}<6 \mathrm{GeV}^{2}$ respectively. The final products $K^{+}, \pi^{-}$associated with the $K^{0 *}$ meson are those with an invariant mass within $\sim 100$ $\mathrm{MeV}$ of the value $m_{K}^{*}=892 \mathrm{MeV}$. (These data correspond to an integrated luminocity of $3 \mathrm{fb}^{-1}$ of proton-proton collisions by LHCb during 2011-2012).

However, as already noted, according to the universality of gauge interactions in the leptonic sector, the SM theoretical predictions are $R_{K}(S M)=R_{K}^{*}(S M) \approx 1$ up to insignificant electromagnetic corrections of $\mathscr{O}\left(m_{\mu} / m_{b}\right)$ [10]. Thus, the measurments of both ratios indicate a deficit in the same direction. Experimentally, in LHCb there are differences regarding the treatment of the decays to a $\mu^{+} \mu^{-}$or $e^{+} e^{-}$final state since these pairs behave differently in their flight through the material of the detector. This is because electrons, being lighter than muons, emit much bremsstralung and as a result there is significant reduction of the momentum, thus the efficiency in the muon channel is much better that in the electron's. This deficiency, however, is improved by a recovery procedure based on the evaluation of the difference between the $p_{T}$ of the $K^{* 0}$ meson and that of the $e^{+} e^{-}$pair, where both are calculated with respect to the direction of the flight of the $B^{0}$ meson (see fig.4).

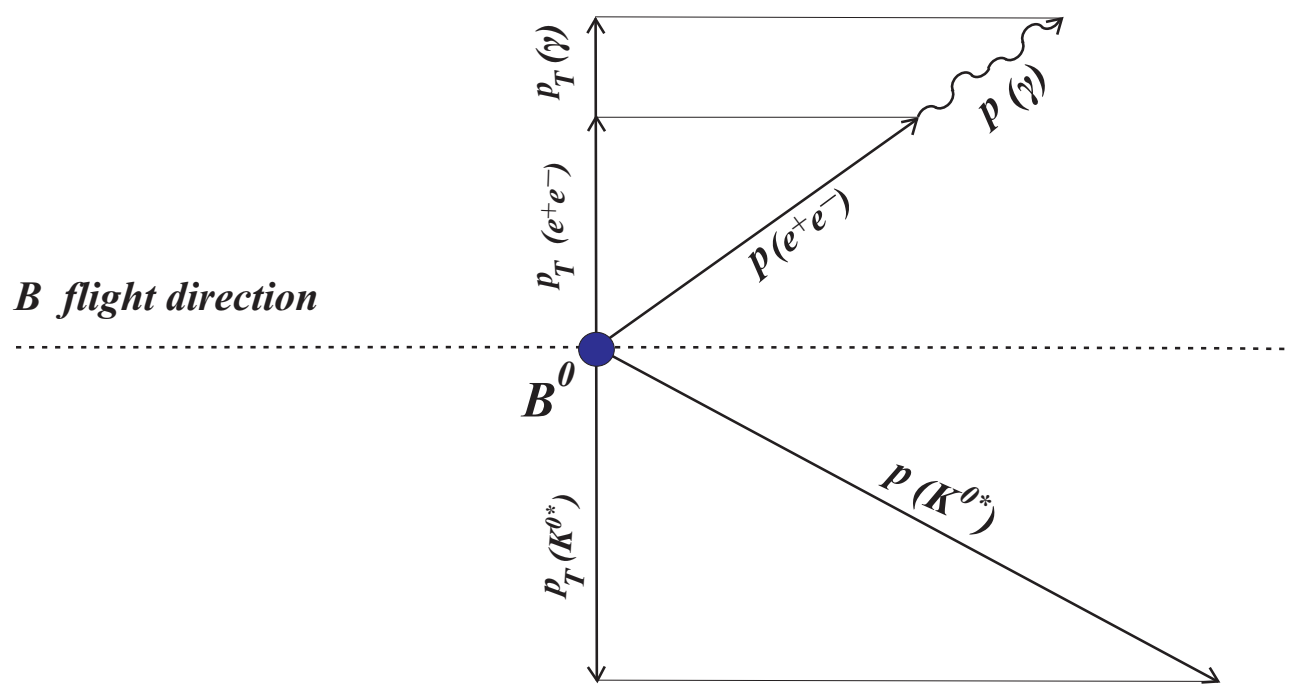

Figure 4: The topology of $B \rightarrow K^{*} e^{+} e^{-}$decay and Bremsstralung effect recovery in accordance to [2]

Eventually, assuming that this experimental evidence remains valid in future analysis, these deficits in the muon channel are unexplained in the Standard Model framework and new physics interactions are required, in order to discriminate the electron from muon ratios in the semileptonic 
$B$ meson decays. Indeed, it is commonly believed that the Standard Model is not the final theory of fundamental interactions, but just an effective low energy limit of some grand unified theory (GUT), possibly embedded in a string scenario. In general, any GUT which includes the SM gauge group predicts new physics phenomena and deviations from the SM predictions. Some common characteristics of a wide class of models derived in such a framework are exotic colour tiplets and singlet fields, new gauge bosons $Z^{\prime}$ associated with additional abelian symmetries etc ${ }^{1}$. All these new ingredients can in principle mediate new exotic processes or enhance others already observed in recent experiments. Since the experimental results of anomalous $B$ meson decays remain in place through the last few years, it is worth exploring viable SM extentions to explain them.

The most popular scenarios to interpret the aforementioned deviations include either exotic leptoquarks, or additional neutral gauge bosons which couple differently to the three fermion families. Given the fact that the composition of mesons in $B \rightarrow K$ decays is $B=u \bar{b}$ and $K=u \bar{s}$, at the fundamental level these results are associated with the $b \rightarrow s \mu^{+} \mu^{-}$transition. The tree-level graphs for such processes are shown in figure 5.
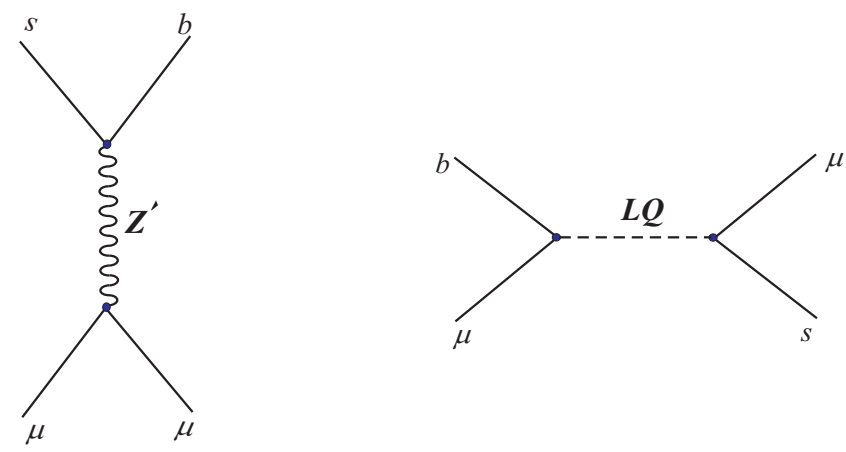

Figure 5: $Z^{\prime}$ boson and leptoquark contributions to $b \rightarrow s \mu^{+} \mu^{-}$

\section{The effective Hamiltonian description}

The contributions from SM physics as well as from any theory beyond the SM, can be described by an effective theory where the heavy degrees of freedom have been integrated out. The relevant Hamiltonian is parametrised in terms of the Wilson coefficients $C_{k}$, which display the strength of the interaction, and a number of quantum operators $\mathscr{O}_{k}$ which encode the Lorentz structure [4]:

$$
\mathscr{H}_{e f f}=-\frac{4 G_{F}}{\sqrt{2}} V_{t b} V_{t s}^{*} \frac{\alpha}{4 \pi} \sum_{k}\left(C_{k}(\mu) \mathscr{O}_{k}(\mu)+C_{k}^{\prime}(\mu) \mathscr{O}_{k}^{\prime}(\mu)\right)
$$

In the above formula the following quantities are involved: $G_{F}=\sqrt{2} /\left(4 v^{2}\right)$ (where $\left.v=174 \mathrm{GeV}\right)$ is the Fermi constant, $V_{t b} \approx 0.95, V_{t s}^{*} \approx 0.4$ are CKM elements relevant to the specific transition, and $\alpha \approx 1 / 128$ is the fine structure constant computed at the scale $m_{b}$. The Wilson coefficients

\footnotetext{
${ }^{1}$ There is a vast number of related papers in the literature. For an incomplete list see [7].
} 
$C_{k}(\mu)$ and the dimension-six quantum operators $\mathscr{O}_{k}(\mu)$ are defined at the scale $\mu=m_{b}$. The Lorenz invariant operators relevant to the processes are

$$
\begin{array}{cc}
\mathscr{O}_{9}=\bar{s} \gamma_{\lambda} P_{L} b \bar{\ell} \gamma^{\lambda} \ell & \mathscr{O}_{9}^{\prime}=\bar{s} \gamma_{\lambda} P_{R} b \bar{\ell} \gamma^{\lambda} \ell \\
\mathscr{O}_{10}=\bar{s} \gamma_{\lambda} P_{L} b \bar{\ell} \gamma^{\lambda} \gamma_{5} \ell & \mathscr{O}_{10}^{\prime}=\bar{s} \gamma_{\lambda} P_{R} b \bar{\ell} \gamma^{\lambda} \gamma_{5} \ell
\end{array}
$$

where $P_{L}=\frac{1}{2}\left(1-\gamma_{5}\right)$ and $P_{R}=\frac{1}{2}\left(1+\gamma_{5}\right)$.

There are several theoretical uncertainties in the computation, the most important come from the form factors and the contributions of the hadronic weak Hamiltonian which emerges from the assumed factorisation of the amplitude into a hadronic and a leptonic part. These are discussed for example in [4]. Focusing in $B^{+} \rightarrow K^{+} \mu^{+} \mu^{-}$in the limit of vanishing lepton mass, the decay rate can be written as

$$
\frac{d \Gamma}{d q^{2}}=\frac{G_{F}^{2} \alpha^{2}}{(4 \pi)^{5} m_{B}^{3}}\left|V_{t b} V_{b s}^{*}\right| g^{3 / 2}\left(m_{B}, m_{K^{*}}, q^{2}\right)\left(\left|F_{V}\right|^{2}+\left|F_{A}\right|^{2}\right)
$$

The quantities in (3.4) are [4]:

$$
g\left(x_{i}\right)=\sum_{i=1}^{3} x_{i}^{2}-2 x_{1} x_{2}-2 x_{2} x_{3}-2 x_{1} x_{3}, F_{V}=\left(C_{9}+C_{9}^{\prime}\right) f_{+}\left(q^{2}\right), F_{A}=\left(C_{10}+C_{10}^{\prime}\right) f_{+}\left(q^{2}\right)+h_{K}
$$

where $f_{+}\left(q^{2}\right)$ is a QCD factor, $h_{K}$ non-factorisable contributions of $\mathscr{H}_{\text {eff }}$, while $C_{7}, C_{7}^{\prime}$ contributions are ignored. As can be seen, there are several operators with different Lorentz structure that could be present in the effective Hamiltonian, but only a few of them are related to the present LHCb data. The correlations of $R_{K} / R_{K^{*}}$ deviations and the corresponding chiral operators generated by New Physics in the $\mu$ sector are plotted in ref [9] and are roughly depicted here in figure 6. The LHCb data can be interpreted assuming a negative contribution to the Wilson coefficient $C_{9}$, with best fit value $C_{9}^{N P} \approx-1.1$. The case of $C_{9}^{N P}=-C_{10}^{N P} \approx-0,5$, which is an $S U(2)_{L}$ invariant solution is also possible. Both solutions ar compatible with negligible values of the Wilson coefficients $C_{9}^{\prime}, C_{10}^{\prime}$.

Let's discuss the case where the data are fit with the first solution. It is, in principle, possible that the required operator is generated from a tree-level process mediated by a $\mathrm{TeV}$ scale $Z^{\prime}$ boson that couples to the $b, s$ quarks and the leptons. This neutral gauge boson can be associated with a new $U(1)^{\prime}$ gauge symmetry spontaneously broken at the $\mathrm{TeV}$ scale. $Z^{\prime}$ couples to a neutral current $L \supset Z^{\prime} \lambda J_{\lambda}^{\prime 0}$ where the couplings to the third quark generation differ from those to the first and second ones. To give an estimate, we use the toy model presented in ref [11], where the current is assumed to be of the form:

$$
\begin{aligned}
J_{\lambda}^{\prime 0}= & g_{t L}\left(\bar{b} \gamma_{\lambda} P_{L} b+\bar{t} \gamma_{\lambda} P_{L} t\right)+g_{\mu}\left(\bar{\mu} \gamma_{\lambda} \mu+\bar{v}_{\mu} \gamma_{\lambda} v_{\mu}\right) \\
& +g_{q L} \sum_{q=u, d, s, c}\left(\bar{q} \gamma_{\lambda} P_{L} q\right)+\left(g_{t L}-g_{q L}\right) V_{t s}^{*} V_{t b} \bar{s} \gamma_{\lambda} P_{L} b+h . c .
\end{aligned}
$$

Then, the new physics contribution to the Wilson coefficient $C_{9}$ is [11]

$$
C_{9}^{N P}=-\frac{\pi g_{\mu}\left(g_{t L}-g_{q L}\right)}{2 \sqrt{2} G_{F} M_{Z^{\prime}}^{2} \alpha \cos ^{2} \theta_{W}} \approx-\frac{\pi g_{\mu}\left(g_{t L}-g_{q L}\right)}{c_{W}^{2}\left(\frac{M_{Z^{\prime}}}{2 \mathrm{TeV}}\right)^{2}}
$$




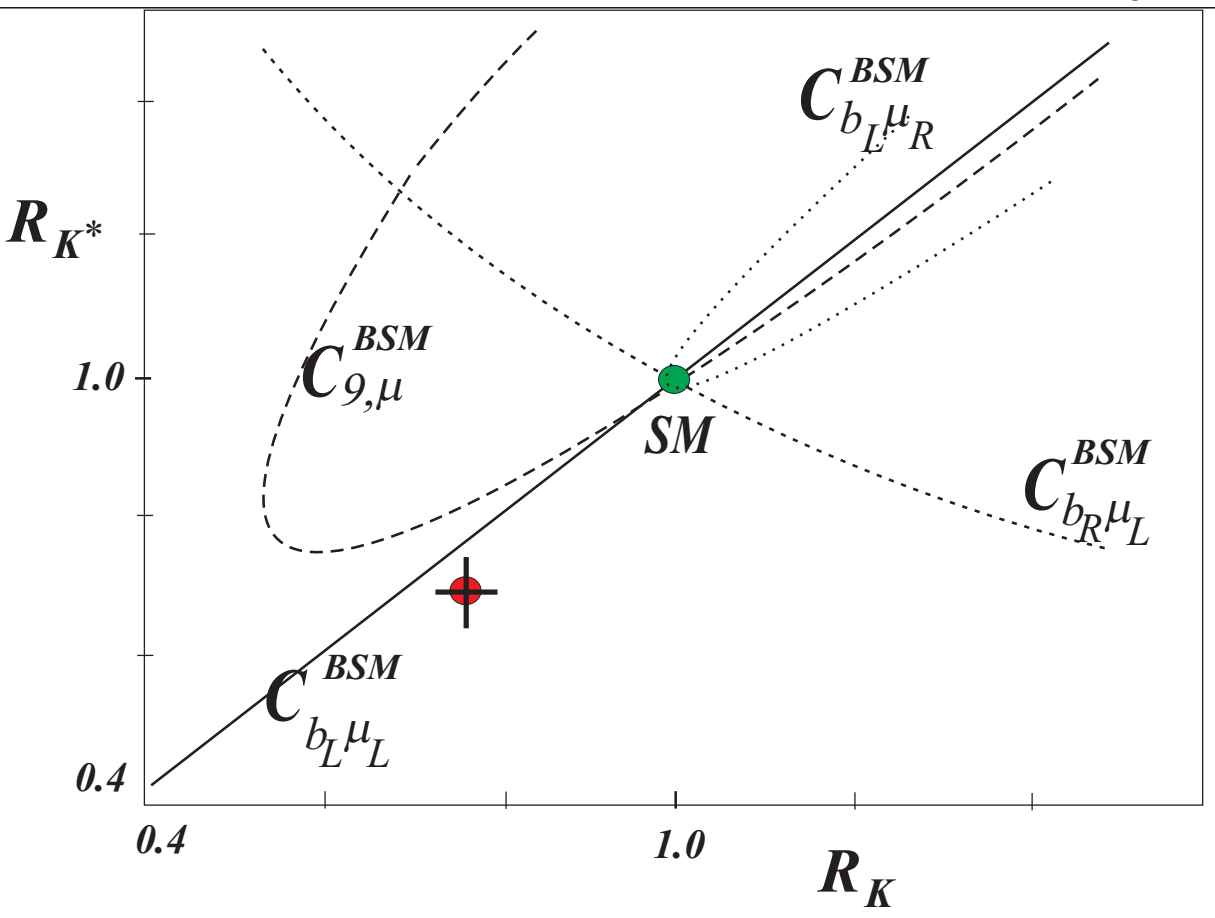

Figure 6: Correlations of $R_{K} / R_{K^{*}}$ for various Lorentz operators. The green dot represents the SM prediction and the red one the experimental value.

For couplings $g_{\alpha} \sim \frac{1}{2}$, and $M_{Z^{\prime}} \sim$ few $\mathrm{TeV}$, ( which is consistent with the renormalisation group

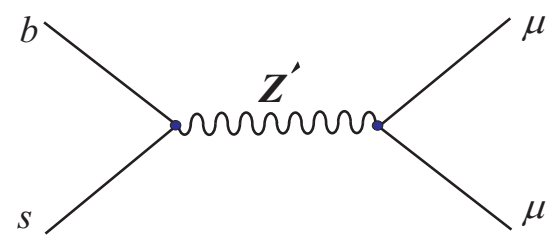

Figure 7: $Z^{\prime}$ boson and $b \rightarrow s \mu \mu$ decay

analysis of models admitting such $U(1)$ symmetries[12]), we can obtain $C_{9}^{N P} \sim-1$ which reduces the total value of $C_{9}$ by $\sim 25 \%$ in accordance with the experimental observations. Notice however, that any theory beyond the SM interpreting the above anomalies must also respect other constraints too, and in particular, with the stringent bounds [13] of Kaon decays such as $\mathscr{B}\left(K^{+} \rightarrow \pi^{+} v \bar{v}\right) \approx$ $17 \pm 10 \times 10^{-11}$ and $\mathscr{B}\left(K_{L} \rightarrow \pi^{0} v \bar{v}\right)<2.6 \times 10^{-8}$.

\section{Non-universal $U(1)$ s in local F-theory models}

A natural way to implement the idea of a new gauge boson $Z^{\prime}$ is within an F-theory framework $[14,15]^{2}$. The so derived model(s) can explain the date either through the existence of a

\footnotetext{
${ }^{2}$ For model building with F-theory and early references see reviews [16, 17, 18]
} 
new light neutral boson coupled differently to families [19], and/or in the presence of a vectorlike family[20, 19]. The interpretation of the data with leptoquarks is also a viable possibility, since plenty of such states appear in a wide class of F-theory models. In this talk, we will focus only on the first case and present a semi-local F-theory $S U(5)$ GUT augmented with a $U(1)^{\prime}$ factor coupled non-universally to leptons, arising from a variant of $E_{6}$ models with $\mathscr{Z}_{2}$ monodromy [21]. We start with the following symmetry breaking,

$$
\begin{aligned}
E_{8} & \supset E_{6} \times U(1)_{\perp}^{\prime} \times U(1)_{\perp} \\
& \supset S O(10) \times U(1)_{\psi} \times U(1)_{\perp}^{\prime} \times U(1)_{\perp} \\
& \supset S U(5)_{G U T} \times U(1)_{\chi} \times U(1)_{\psi} \times U(1)_{\perp}^{\prime} \times U(1)_{\perp},
\end{aligned}
$$

Imposing a $\mathscr{Z}_{2}$ monodromy, the symmetry of the model reduces to $S U(5)_{G U T} \times U(1)^{3}$ and at the same time a top Yukawa coupling is allowed at tree-level[15]. We choose the following basis for the Cartan generators corresponding to the three remaining abelian factors:

$$
Q_{\perp}=\frac{1}{2 \sqrt{3}} \operatorname{diag}(1,1,-2,0,0), Q_{\psi}=\frac{1}{2 \sqrt{6}} \operatorname{diag}(1,1,1,-3,0), Q_{\chi}=\frac{1}{2 \sqrt{10}} \operatorname{diag}(1,1,1,1,-4) .
$$

Next, since we would like to explain the experimental results invoking the existence of a TeV scale neural gauge boson $Z^{\prime}$, we assume that a low energy $U(1)^{\prime}$ is generated by a linear combination of the unbroken $U(1)$ 's:

$$
Q^{\prime}=c_{1} Q_{\perp}+c_{2} Q_{\psi}+c_{3} Q_{\chi}
$$

Furthermore, in order to retain $S U(5)_{\perp}$ normalisation, the coefficients $c_{i}$ are subject to the condition

$$
c_{1}^{2}+c_{2}^{2}+c_{3}^{2}=1
$$

An effective $S U(5)$ model now can be constructed by assuming suitable fluxes along the $U(1)$ factors. These fluxes generate chirality for the $10 / \overline{10}$ and $5 / \overline{5}$ representations residing in the intersections (matter curves) of the $S U(5)$ GUT divisor and the 7-branes associated with the abelian factors. There are initially ten matter curves available to accommodate the fiveplets and five matter curves for the tenplets but after the $\mathscr{Z}_{2}$ monodromy action they reduce to seven and four respectively $[22,23]$. We designate the corresponding numbers of the $S U(5)$ multiplets on the matter curves with $M_{5_{i}}, M_{10_{j}}$ respectively. Furthermore, we may turn on flux along the hypercharge generator $U(1)_{Y}$ which breaks $S U(5)$ down to $\mathrm{SM}$ and at the same time splits the $10, \overline{10}$ and $5, \overline{5}$ 's into different numbers of Standard Model multiplets. Parametrising the hyperfluxes with the integers $N_{7,8,9}$ and assuming a linear combination of them, $N_{y}$, to be the hyperflux piercing a given matter curve, the 10-plets and 5-plets split according to the following pattern

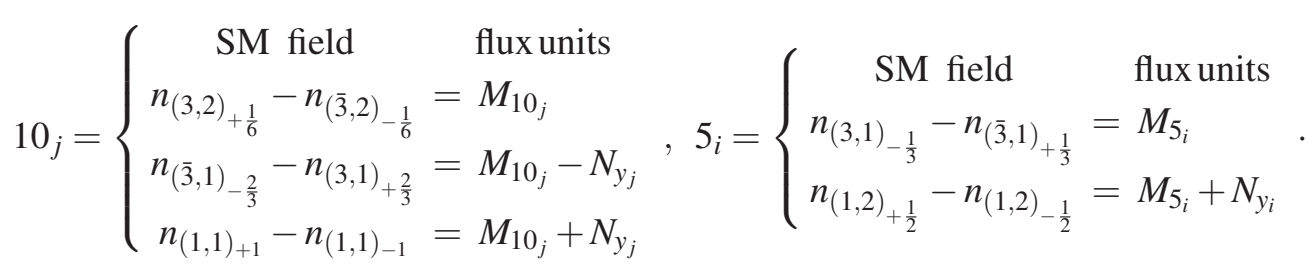




\begin{tabular}{|c|c|c|c|c|c|c|c|c|c|c|c|c|c|c|}
\hline$c_{1}$ & $c_{2}$ & $c_{3}$ & $M_{5_{H_{u}}}$ & $M_{5_{1}}$ & $M_{5_{2}}$ & $M_{5_{3}}$ & $M_{5_{4}}$ & $M_{5_{5}}$ & $M_{5_{6}}$ & $M_{10_{t}}$ & $M_{10_{2}}$ & $M_{10_{3}}$ & $M_{10_{4}}$ & $N_{7,8,9}$ \\
\hline 0 & $\frac{\sqrt{\frac{15}{34}}}{2}$ & $\frac{11}{2 \sqrt{34}}$ & 0 & 0 & 0 & 0 & -1 & -3 & 1 & 1 & 2 & -1 & 1 & $N_{7}=1$ \\
$-\frac{\sqrt{\frac{5}{6}}}{2}$ & $-\frac{5 \sqrt{\frac{5}{3}}}{8}$ & $\frac{3}{8}$ & 0 & 1 & -1 & 0 & -1 & -2 & 0 & 2 & -1 & 1 & 1 & $N_{9}=1$ \\
$\frac{\sqrt{3}}{2}$ & $-\sqrt{\frac{3}{32}}$ & $\sqrt{\frac{5}{32}}$ & 0 & 0 & 0 & 1 & -3 & -1 & 0 & 2 & 1 & 1 & -1 & $N_{8}=1$ \\
\hline
\end{tabular}

Table 1: Flux parameters and $c_{i}$ coefficients for three models

\begin{tabular}{|c|c|c|c|c|c|c|}
\hline & & Model A & \multicolumn{2}{|c|}{ Model B } & \multicolumn{2}{|r|}{ Model C } \\
\hline Curve & $Q^{\prime} \sqrt{85}$ & SM Content & $Q^{\prime} \sqrt{10}$ & SM Content & $Q^{\prime}$ & SM Content \\
\hline \multirow{2}{*}{$5_{H_{u}}$} & -4 & $H_{u}$ & $\frac{3}{2}$ & $H_{u}$ & $-\frac{1}{2}$ & $H_{u}$ \\
\hline & - & - & - & - & - & - \\
\hline \multirow{2}{*}{$5_{1}$} & - & - & $\frac{1}{4}$ & $\overline{d^{c}}$ & - & - \\
\hline & 4 & $H_{d}$ & - & - & $-\frac{1}{4}$ & $L$ \\
\hline \multirow{2}{*}{$5_{2}$} & - & - & - & - & - & - \\
\hline & $\frac{3}{2}$ & $L$ & 1 & $d^{c}+2 L$ & $\frac{1}{2}$ & $H_{d}$ \\
\hline \multirow{2}{*}{$5_{3}$} & - & - & - & - & 0 & $\overline{d^{c}}$ \\
\hline & $-\frac{7}{2}$ & $L$ & $-\frac{3}{2}$ & $H_{d}$ & - & - \\
\hline \multirow{2}{*}{$5_{4}$} & - & - & - & - & - & - \\
\hline & $\frac{3}{2}$ & $d^{c}$ & $\frac{9}{4}$ & $d^{c}+L$ & $-\frac{1}{4}$ & $3 d^{c}+2 L$ \\
\hline \multirow{2}{*}{$5_{5}$} & - & - & - & - & - & - \\
\hline & $-\frac{7}{2}$ & $3 d^{c}+2 L$ & $-\frac{1}{4}$ & $2 d^{c}+L$ & $-\frac{3}{4}$ & $d^{c}+L$ \\
\hline \multirow{2}{*}{$5_{6}$} & 6 & $\overline{d^{c}}+\bar{L}$ & -1 & $\bar{L}$ & $0^{4}$ & $\bar{L}$ \\
\hline & - & - & - & - & - & - \\
\hline \multirow{2}{*}{$10_{t}$} & 2 & $Q+2 u^{c}$ & $-\frac{3}{4}$ & $2 Q+3 u^{c}+e^{c}$ & $\frac{1}{4}$ & $2 Q+3 u^{c}+e^{c}$ \\
\hline & - & - & - & - & - & - \\
\hline \multirow{2}{*}{$10_{2}$} & 2 & $2 Q+u^{c}+3 e^{c}$ & - & - & $-\frac{1}{2}$ & $Q+u^{c}+e^{c}$ \\
\hline & - & - & $-\frac{1}{2}$ & $\bar{Q}+\overline{u^{c}}+\overline{e^{c}}$ & - & - \\
\hline \multirow{2}{*}{$10_{3}$} & - & - & $\frac{7^{2}}{4}$ & $Q+u^{c}+e^{c}$ & $\frac{1}{4}$ & $Q+2 e^{c}$ \\
\hline & $\frac{1}{2}$ & $\bar{Q}+\overline{u^{c}}+\overline{e^{c}}$ & - & - & - & - \\
\hline \multirow{2}{*}{$10_{4}$} & $\frac{11}{2}$ & $Q+u^{c}+e^{c}$ & $-\frac{3}{4}$ & $Q+2 e^{c}$ & - & - \\
\hline & - & - & - & - & $\frac{1}{4}$ & $\bar{Q}+\overline{u^{c}}+\overline{e^{c}}$ \\
\hline
\end{tabular}

Table 2: The low energy spectrum for the three models of Table 1 . (There are also singlet fields [19] not shown in this table)

The integers $M_{10_{j}}, M_{5_{i}}$ representing the multiplicities and the coefficients $c_{k}$ defining the linear combination (4.3) are subject to anomaly cancellation conditions [19] (see also related work for $B$-decays [24]) and the constraint (4.4) respectively. There are numerous solutions [19] to these constraints. Three examples are given in Table 1 where the flux data and solutions for the coefficients $c_{i}$ are presented. The spectrum of these three models is determined by the integers $M_{i}, N_{j}$ which are computed in consistency with the anomaly cancellation conditions and can be seen in Table 2. We also mention the existence of several singlet fields in the spectrum which may acquire non-zero vevs and, amongst other things, they play a crucial rôle in the anomaly cancellation conditions and the Yukawa couplings of the effective theory [19]. 
From Table 2 we see that model $C$ has 3 chiral families and a vectorlike one. A model consistent with all the experimental constraints requires a careful distribution of the chiral particle content on the various matter curves. Thus, in view of the stringent experimental constraints from the $K^{0}-\bar{K}^{0}$ oscillations, the three families are accommodated so that their left-handed quark doublets have equal charges, $Q^{\prime}=\frac{1}{4}$, with respect to $U(1)^{\prime}$. Also, the three down quarks and lepton doublets are distributed in 'curves' of fiveplets with the same charge $Q^{\prime}=-\frac{1}{4}$, in Table 2. We may accommodate the vectorlike pair of lepton doublets on the curves $5_{5,6}$ which have non-universal $U(1)^{\prime}$ charges and can mix differently with the lepton doublets $L_{1,2}$, inducing non-universal couplings in the physical left-handed electrons and muons. This can account for the observed ratios $R_{K}$ and $R_{K^{*}}$ in $B$ decays and it is discussed in detail in [20] and [19].

In conclusion, we have presented a class of models with $S U(5) \times U(1)^{\prime}$ gauge symmetry embedded in $E_{8} \supset S U(5) \times U(1)_{\perp}^{4}$. The abelian factor $U(1)^{\prime}$ is a linear combination of the three remaining $U(1) \perp$ factors, after a monodromy is implemented and the corresponding gauge boson $Z^{\prime}$ displays non-universal gauge couplings to fermion families. From class $C$ of Table 2, a phenomenologically promising model emerges with universal couplings to the three chiral fermion generations and only one single vectorlike family having non-universal couplings. Their couplings modify the branching ratios of the $B$-decays in accordance with the recently observed $\mu^{+} \mu^{-}$ deficit.

The author would like to thank the organisers of the "Corfu Summer Institute" for kind hospitality

\section{References}

[1] R. Aaij et al. [LHCb Collaboration], Phys. Rev. Lett. 113 (2014) 151601 doi:10.1103/PhysRevLett.113.151601 [arXiv:1406.6482].

[2] R. Aaij et al. [LHCb Collaboration], arXiv:1705.05802.

[3] Monica Pepe Altarelli, "Rare decays and searcher for NP at LHCb", in Workshop on the Standard Model and Beyond, Corfu 2-10 September, 2017. See also talks of S.F. King, A. Crivellin and R. Zwicky.

[4] W. Altmannshofer and D. M. Straub, Eur. Phys. J. C 75 (2015) no.8, 382 doi:10.1140/epjc/s10052-015-3602-7 [arXiv:1411.3161].

[5] F. Archilli, M.-O. Bettler, P. Owen, K. A. Petridis, Nature volume 546, page 221 (08 June 2017) doi:10.1038/nature21721

[6] Y. Grossman and P. Tanedo, arXiv:1711.03624.

[7] S. Descotes-Genon, J. Matias, M. Ramon and J. Virto, JHEP 1301 (2013) 048 doi:10.1007/JHEP01(2013)048 [arXiv:1207.2753]. C. Bobeth, G. Hiller and D. van Dyk, Phys. Rev. D 87 (2013) no.3, 034016 [Phys. Rev. D 87 (2013) 034016] doi:10.1103/PhysRevD.87.034016 [arXiv:1212.2321] . S. Descotes-Genon, J. Matias and J. Virto, Phys. Rev. D 88 (2013) 074002 doi:10.1103/PhysRevD.88.074002 [arXiv:1307.5683]. S. Jäger and J. Martin Camalich, JHEP 1305 (2013) 043 doi:10.1007/JHEP05(2013)043 [arXiv:1212.2263]. W. Altmannshofer, P. Ball,

A. Bharucha, A. J. Buras, D. M. Straub and M. Wick, JHEP 0901 (2009) 019 
doi:10.1088/1126-6708/2009/01/019 [arXiv:0811.1214]. S. L. Glashow, D. Guadagnoli and K. Lane, Phys. Rev. Lett. 114 (2015) 091801 doi:10.1103/PhysRevLett.114.091801 [arXiv:1411.0565].

R. Gauld, F. Goertz and U. Haisch, Phys. Rev. D 89 (2014) 015005 doi:10.1103/PhysRevD.89.015005 [arXiv:1308.1959]. G. Hiller and M. Schmaltz, Phys. Rev. D 90 (2014) 054014 doi:10.1103/PhysRevD.90.054014 [arXiv:1408.1627]. A. Crivellin, G. D’Ambrosio and J. Heeck, Phys. Rev. Lett. 114 (2015) 151801 doi:10.1103/PhysRevLett.114.151801 [arXiv:1501.00993]. T. Hurth, F. Mahmoudi and S. Neshatpour, JHEP 1412 (2014) 053 doi:10.1007/JHEP12(2014)053 [arXiv:1410.4545]. J. Lyon and R. Zwicky, Phys. Rev. D 88 (2013) no.9, 094004 doi:10.1103/PhysRevD.88.094004 [arXiv:1305.4797].

[8] F. Goertz, J. F. Kamenik, A. Katz and M. Nardecchia, JHEP 1605 (2016) 187 doi:10.1007/JHEP05(2016)187 [arXiv:1512.08500]. C. Bonilla, T. Modak, R. Srivastava and J. W. F. Valle, arXiv:1705.00915 .

[9] G. D'Amico, M. Nardecchia, P. Panci, F. Sannino, A. Strumia, R. Torre and A. Urbano, JHEP 1709 (2017) 010 doi:10.1007/JHEP09(2017)010 [arXiv:1704.05438 ].

[10] G. Hiller and F. Kruger, Phys. Rev. D 69 (2004) 074020 doi:10.1103/PhysRevD.69.074020 [hep-ph/0310219].

[11] B. Allanach, F. S. Queiroz, A. Strumia and S. Sun, Phys. Rev. D 93 (2016) no.5, 055045 Erratum: [Phys. Rev. D 95 (2017) no.11, 119902] doi:10.1103/PhysRevD.93.055045, 10.1103/PhysRevD.95.119902 [arXiv:1511.07447 ].

[12] A. Karozas, G. K. Leontaris and Q. Shafi, Phys. Lett. B 778 (2018) 213 doi:10.1016/j.physletb.2018.01.032 [arXiv:1710.00929 [hep-ph]].

[13] A. J. Buras, D. Buttazzo, J. Girrbach-Noe and R. Knegjens, JHEP 1511 (2015) 033 doi:10.1007/JHEP11(2015)033 [arXiv:1503.02693 [hep-ph]].

[14] C. Beasley, J. J. Heckman and C. Vafa, JHEP 0901 (2009) 058 doi:10.1088/1126-6708/2009/01/058 [arXiv:0802.3391 [hep-th]].

[15] V. Bouchard, J. J. Heckman, J. Seo and C. Vafa, JHEP 1001 (2010) 061 doi:10.1007/JHEP01(2010)061 [arXiv:0904.1419 [hep-ph]].

[16] J. J. Heckman, Ann. Rev. Nucl. Part. Sci. 60 (2010) 237 doi:10.1146/annurev.nucl.012809.104532 [arXiv:1001.0577].

[17] G. K. Leontaris, PoS CORFU 2011 (2011) 095 [arXiv:1203.6277].

[18] A. Maharana and E. Palti, Int. J. Mod. Phys. A 28 (2013) 1330005 doi:10.1142/S0217751X13300056 [arXiv:1212.0555].

[19] M. C. Romao, S. F. King and G. K. Leontaris, arXiv:1710.02349 [hep-ph].

[20] S. F. King, JHEP 1708, 019 (2017) doi:10.1007/JHEP08(2017)019 [arXiv:1706.06100 [hep-ph]].

[21] J. C. Callaghan, S. F. King, G. K. Leontaris and G. G. Ross, JHEP 1204 (2012) 094 [arXiv:1109.1399 [hep-ph]], J. C. Callaghan and S. F. King, JHEP 1304 (2013) 034 doi:10.1007/JHEP04(2013)034 [arXiv:1210.6913 [hep-ph]]. J. C. Callaghan, S. F. King and G. K. Leontaris, JHEP 1312 (2013) 037 doi:10.1007/JHEP12(2013)037 [arXiv:1307.4593 [hep-ph]].

[22] E. Dudas and E. Palti, JHEP 1001 (2010) 127 doi:10.1007/JHEP01(2010)127 [arXiv:0912.0853]. 
[23] S. F. King, G. K. Leontaris and G. G. Ross, Nucl. Phys. B 838 (2010) 119 doi:10.1016/j.nuclphysb.2010.05.014 [arXiv:1005.1025 [hep-ph]].

[24] J. Ellis, M. Fairbairn and P. Tunney, arXiv:1705.03447 [hep-ph]. 